\title{
Clinical Characteristics of 852 Patients with Subacute Thyroiditis before Treatment
}

\author{
Eijun Nishihara, Hidemi Ohye, Nobuyuki Amino, Kazuna Takata, Takeshi Arishima, \\ Takumi Kudo, Mitsuru Ito, Sumihisa Kubota, Shuji Fukata and Akira Miyauchi
}

\begin{abstract}
Objective Subacute thyroiditis (SAT) is a transient inflammatory disease of the thyroid. We evaluated the clinical characteristics based on laboratory and imaging studies in patients with SAT before treatment.

Patients We reviewed the medical records of 852 patients (107 men and 745 women) with SAT who visited our thyroid clinic at Kuma Hospital from 1996 through 2004.

Results SAT developed most often in female patients aged 40 to 50 years, with significant seasonal clusters during summer to early autumn. While the rates of any virus infections and diseases did not differ from those in the general population, recurrent episodes of SAT at intervals of $13.6 \pm 5.6$ years accounted for $1.6 \%$ of all cases. At the onset of SAT, $28.2 \%$ of patients had temperatures greater than $38^{\circ} \mathrm{C}$ and typical symptoms associated with thyrotoxicosis developed in more than $60 \%$ of patients. Before treatment, most of the abnormal laboratory findings associated with thyrotoxicosis, inflammation, and liver dysfunction reached peak levels within 1 week after onset. Ultrasound examination showed that half of the patients with unilateral thyroid pain presented with bilateral hypoechogenic area in the thyroid and the rate of bilateral hypoechogenic area tended to increase 2 months after onset.

Conclusion Laboratory studies of thyroid dysfunction and inflammation related to SAT presented peak levels within 1 week after onset.
\end{abstract}

Key words: subacute thyroiditis, seasonal cluster, thyrotoxicosis, recurrence, hypoechogenic area

(Inter Med 47: 725-729, 2008)

(DOI: 10.2169/internalmedicine.47.0740)

\section{Introduction}

Subacute thyroiditis (SAT) is a self-limiting inflammatory disease of the thyroid. Compared with Graves' disease and Hashimoto's thyroiditis, SAT is uncommon, occurring at the rate of about 1 case per 5 cases of Graves' disease and 1 case per 15 or 20 cases of Hashimoto's thyroiditis (1). The first description of SAT was in 1895 by Mygind (2) and the etiology is still unclear. Although a viral infection has most often been implicated as the cause of SAT, the evidence is generally indirect, and includes a preceding upper respiratory tract infection, the presence of elevated titers of several viral antibodies in the sera, and seasonal and geographic case clusters. The histocompatibility antigen (HLA) studies show that approximately two-thirds of patients with SAT have HLA B35 (3), suggesting that the susceptibility to SAT is genetically influenced. The characteristics features of SAT are acute onset of pain in the thyroid region and various symptoms associated with thyrotoxicosis. A few large scale studies have been reported to characterize laboratory and imaging findings at onset in patients with SAT (4-6). In this study, we reviewed the clinical courses of 852 subjects with SAT through background factors, laboratory and imaging studies.

\section{Subjects and Methods}

\section{Subjects}

The subjects were 852 patients (107 men and 745 women) with SAT who visited our thyroid clinic at Kuma 
Hospital from 1996 through 2004. The diagnosis of SAT was based on clinical features of swelling with pain and tenderness in the thyroid gland, laboratory findings of elevated $\mathrm{C}$-reactive protein (CRP), elevated serum free thyroxine (FT 4) and decreased serum thyroid stimulating hormone (TSH), or suppressed 24-hour radioactive iodine uptake (RAIU). Anti-thyroid autoantibodies were negative or weakly positive. All patients with SAT showed characteristic features of hypoechogenic area (HEA) at a painful portion of the thyroid gland by ultrasonography. The clinical signs and symptoms in patients with SAT were recorded based on the information of unified interview sheets. The SAT onset was defined as the point of a swelling with pain and tenderness in the thyroid gland.

\section{Laboratory evaluation}

Serum levels of aspartate aminotransferase (AST; normal range, 5 to $40 \mathrm{IU} / \mathrm{l}$ ), alanine aminotransferase (ALT; normal range, 5 to $35 \mathrm{IU} / \mathrm{l}$ ), and CRP (normal range less than 0.5 $\mathrm{mg} / \mathrm{dl}$ ) were measured using a routine automated analyzer (Hitachi 7170S Clinical Analyzer, Tokyo, Japan). Concentrations of serum TSH, FT4, and free triiodothyronine (FT3) were measured with enzyme immunoassays (AxSYM TSH, AxSYM FT4, and AxSYM FT3, respectively, Abbott Japan Co., Tokyo, Japan). The reference ranges used for serum TSH, FT4 and FT3 were 0.30-5.00 mIU/l, 1.70-3.70 pg/ml and $0.70-1.60 \mathrm{ng} / \mathrm{dl}$, respectively. Serum thyroglobulin $(\mathrm{Tg}$; normal range less than $35 \mathrm{mg} / \mathrm{dl}$ ) was measured with an enzyme-linked immunosorbent assay. Other than a change in the lower limit of detection of serum TSH, despite some changes in assay methods, the reference ranges did not change during the study period.

\section{Statistical analysis}

Comparisons of variables among different groups were performed with the Tukey-Kramer multiple comparison test. The $\chi^{2}$ test was used for statistical analysis of data in Fig. 1B and Table 1. Data are reported as mean \pm SD. A level of $\mathrm{p}<0.05$ was accepted as indicating statistical significance.

Results

\section{Clinical features at onset}

There were 852 patients with SAT from 1996 through 2004: the female-male ratio was $7: 1$ and mean age was $47.8 \pm 9.4$ years (range, 22-83 years; median, 48 years) (Fig. 1A). The distribution of the month of SAT onset is shown in Fig. 1B. SAT developed in $44 \%$ of patients from July through October, and this cluster from summer to early autumn was significantly different from other seasonal clusters $\left(\chi^{2}=46.8 ; \mathrm{p}<0.001\right)$.

At the onset of SAT, $68.2 \%$ of patients developed neck pain in a unilateral side of the thyroid and others developed neck pain in the bilateral side. Aside from neck pain, symptoms related to upper respiratory infection, including nasal discharge, cough and sputum, were noted in $23 \%$ of patients within 1 month before the onset of SAT, and $28.2 \%$ of patients had temperatures of greater than $38^{\circ} \mathrm{C}$ (Fig. 1C). Typical symptoms associated with thyrotoxicosis, including palpitations, increased sweating, and weight loss, developed in $62.1 \%$ of patients (Fig. 1C). Figure 2A shows the rates of diseases occurring in more than $1 \%$ of patients. The rates of any virus infections and diseases did not differ from those in the general population. Recurrent episodes of SAT accounted for $1.6 \%$ of all cases, with an interval of 13.6 \pm 5.6 years between the first and the second episodes (Fig. 2A, B). Anti-thyroid autoantibodies were negative in these recurrent patients.

\section{Comparison of initial laboratory and imaging find- ings in patients with SAT}

Of the 852 patients, $96.4 \%$ of patients visited our hospital less than 45 days after the onset of neck pain. To evaluate the acute phase of SAT before treatment according to the interval from the onset, we compared the initial laboratory and imaging studies of three groups (less than 7 days, 30 \pm 5 days, and more than 60 days) separately (Table 1). The TSH level was significantly lower $(\mathrm{p}<0.05)$ in patients examined 1 month after onset than in patients examined within 7 days and the percentages of undetectable TSH concentrations in the three groups were $52.3 \%, 64.5 \%$, and $40.0 \%$, respectively. However, the levels of FT3, FT4, white blood cells (WBC), CRP, Tg, and RAIU did not differ significantly between the three groups. The levels of aminotransferases did not differ significantly between the groups, but patients with higher than normal ALT levels in the three groups were $25.7 \%, 23.5 \%$, and $15.7 \%$, respectively. Of the patients examined less than 7 days after onset, $26.2 \%$ had neck pain on both sides of the thyroid, and $54.8 \%$ had bilateral HEA in the thyroid on ultrasound examination (Table 1). The rate of bilateral HEA tended to increase with time after onset of pain, but the $\chi^{2}$ test showed no significant difference in the prevalence of HEAs between patients examined among the three groups $\left(\chi^{2}=8.04 ; \mathrm{p}=0.09\right)$.

Discussion

In this study, we evaluated the characteristics of SAT at onset in 852 patients living in Kobe and neighboring areas. Most subjects were women aged 40 to 50 years, and significant seasonal clusters of cases developed during the summer and early autumn. SAT has been reported most commonly in the areas having four definite seasons in North America, Europe, and Japan, and predominance in this season has also been reported in similar temperature zones (7-9). Aside from neck pain, symptoms associated with thyrotoxicosis were more frequently observed than symptoms related to upper respiratory tract infection throughout the clinical course of SAT.

An association between SAT and HLA B35 has been noted in all ethnic groups tested $(3,10-12)$. Familial occur- 

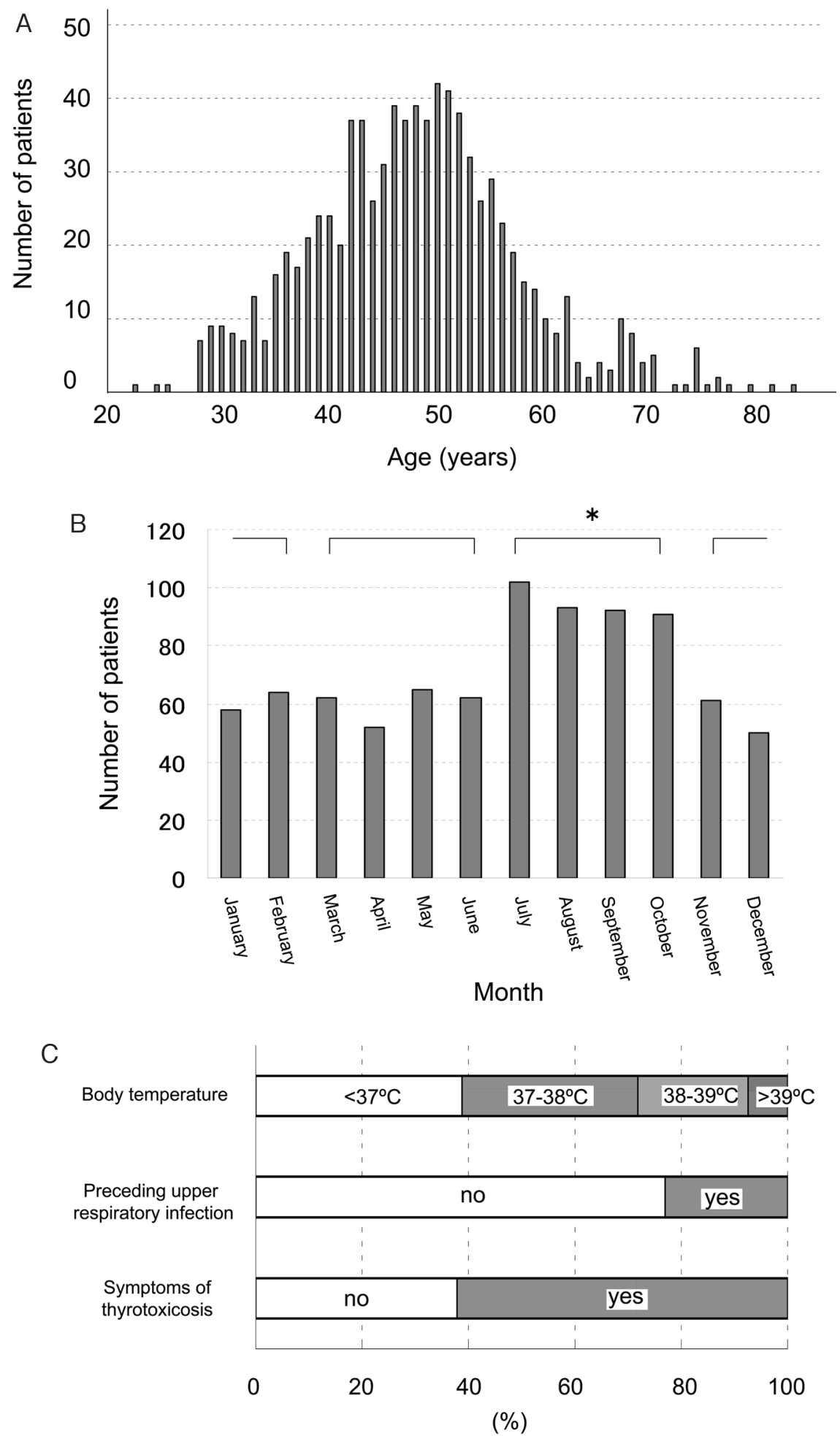

Figure 1. (A) Age distribution of patients with SAT. (B) Seasonal distribution of SAT onset. Asterisk indicates significant difference $(\mathbf{p}<\mathbf{0 . 0 0 1})$ among the three seasonal clusters. $(\mathrm{C})$ Clinical features at onset of SAT.

rence of SAT with HLA B35 has also been reported (1315). This evidence suggests that SAT might occur in genetically predisposed individuals. The history of patients showed no obvious association between SAT and other diseases, including virus infection. The titers of various viral antibodies were not increased during recurrence (16). These findings suggest that if associated with viral infection, the recurrence of SAT seems to occur not under continuous viral infection but after the disappearance of immunity from a previous viral infection. Recurrence after recovery is rare, but SAT recurred in $1.6 \%$ of patients, with a rate and interval similar to those in previous reports $(4,16,17)$. Another report (17) and our data suggested that the presence of antithyroid autoantibodies was not correlated with the recurrent 

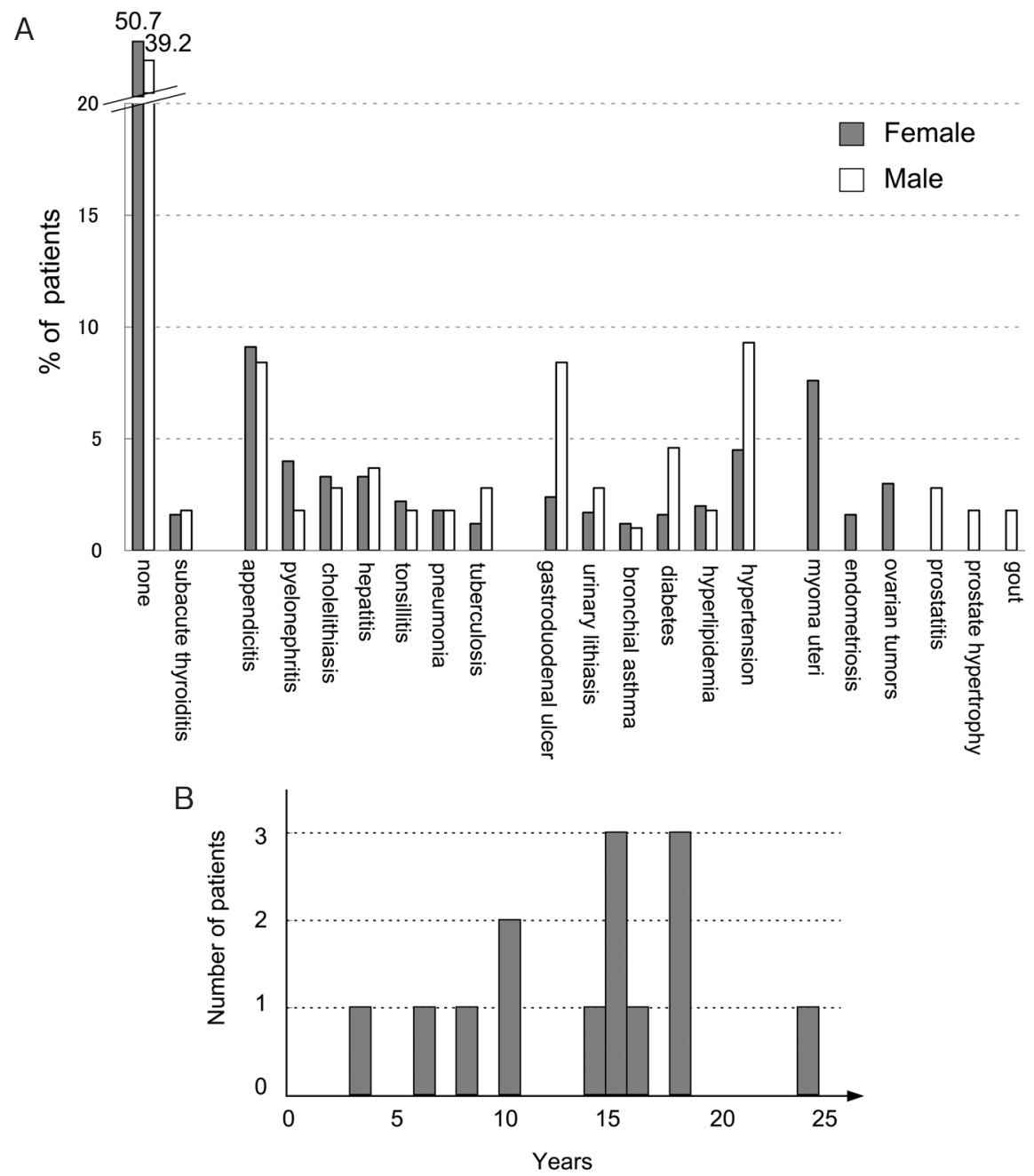

Figure 2. (A) Past history of patients with SAT. The rate of each condition is greater than $1 \%$. (B) The interval and frequencies of recurrence from the previous episode of SAT.

Table 1. Comparison of Initial Data in Patients with SATClassified According to the Interval from the Onset of Pain

\begin{tabular}{|c|c|c|c|}
\hline & $\begin{array}{c}<7 \text { days } \\
(n=42)\end{array}$ & $\begin{array}{c}30 \pm 5 \text { days } \\
(\mathrm{n}=282)\end{array}$ & $\begin{array}{l}>60 \text { days } \\
(n=20)\end{array}$ \\
\hline FT4 (ng/dl) & $2.51 \pm 1.15$ & $2.36 \pm 1.05$ & $2.28 \pm 1.35$ \\
\hline FT3 (pg/ml) & $5.90 \pm 2.99$ & $5.85 \pm 2.60$ & $4.66 \pm 1.24$ \\
\hline FT3/ FT4 & $2.31 \pm 0.82$ & $2.55 \pm 1.54$ & $2.28 \pm 0.66$ \\
\hline $\mathrm{TSH}(\mathrm{IU} / \mathrm{ml})$ & $0.073 \pm 0.18$ & $0.029 \pm 0.11^{\mathrm{a}}$ & $0.008 \pm 0.012$ \\
\hline RAIU $(\%)^{b}$ & $2.02 \pm 3.82$ & $1.20 \pm 1.40$ & $1.29 \pm 0.96$ \\
\hline WBC $(/ \mu \mathrm{l})$ & $6449 \pm 1501$ & $6598 \pm 1904$ & $5980 \pm 903.5$ \\
\hline CRP (mg/dl) & $3.52 \pm 2.81$ & $3.16 \pm 3.34$ & $2.93 \pm 2.68$ \\
\hline $\operatorname{Tg}(\mathrm{ng} / \mathrm{ml})$ & $597 \pm 853$ & $478 \pm 948$ & $206 \pm 166$ \\
\hline ALT (IU/I) & $27.3 \pm 18.6$ & $31.0 \pm 32.0$ & $29.6 \pm 23.0$ \\
\hline AST (IU/I) & $24.3 \pm 16.9$ & $23.8 \pm 20.9$ & $21.5 \pm 10.4$ \\
\hline HEA (\%) & & & \\
\hline Bilateral & 54.8 & 75.5 & 90.0 \\
\hline Right lobe & 28.6 & 16.0 & 10.0 \\
\hline Left lobe & 16.6 & 8.5 & 0.0 \\
\hline
\end{tabular}

${ }^{a} \mathrm{p}<0.05$ compared to the group of less than 7 days ${ }^{b}$ Normal range, $10-40 \% / 24 \mathrm{~h}$ episode.

We found that abnormal laboratory findings, such as elevations of FT4, FT3, Tg, CRP, and aminotransferases, reached their peak levels within 7 days after onset, whereas fully suppressed TSH could be detected later, in agreement with a previous report (18). The duration of elevated FT4 was much longer in SAT than in other types of destructive thyroiditis, including painless thyroiditis and acute suppurative thyroiditis, whereas the duration and levels of liver dysfunction in SAT were similar to those in painless thyroiditis $(19,20)$. Importantly, half of patients with unilateral thyroid pain had HEA on the uninvolved side of the thyroid within 7 days from onset, suggesting that ultrasound examination is sensitive for early bilateral inflammatory changes. Moreover, increased bilateral HEA with time after onset implied spreading inflammatory changes toward the uninvolved side of the thyroid even under decreased levels of serum CRP.

Collectively, retrospective studies to characterize SAT onset in a large scale showed significant seasonal clusters and $1.6 \%$ of SAT recurrent episodes. Most of the laboratory findings of thyroid dysfunction and inflammation caused by SAT presented peak levels within 1 week after onset, and ultrasound examination was sensitive to confirm early bilat- 
eral inflammatory changes in the thyroid at onset.

\section{Acknowledgement}

This work was supported in part by Medical Research Fund of Hyogo Medical Association (MRF-H-08-05).

\section{References}

1. Guimaraes V. Subacute and Riedel's Thyroiditis. In: Endocrinology. 5th ed. DeGroot LJ, Jameson JL, et al, Eds. Elsevier, Philadelphia, 2006: 2069-2078.

2. Mygind H. Thyroiditis akuta simplex. J Laryngol 91: 181-193, 1895.

3. Nyulassy S, Hnilica P, Buc M, Guman M, Hirschova V, Stefanovic J. Subacute (de Quervain's) thyroiditis: association with HLA-Bw35 antigen and abnormalities of the complement system, immunoglobulins and other serum proteins. J Clin Endocrinol Metab 45: 270-274, 1977.

4. Fatourechi V, Aniszewski JP, Fatourechi GZ, Atkinson EJ, Jacobsen SJ. Clinical features and outcome of subacute thyroiditis in an incidence cohort: Olmsted County, Minnesota, study. J Clin Endocrinol Metab 88: 2100-2105, 2003.

5. Benbassat CA, Olchovsky D, Tsvetov G, Shimon I. Subacute thyroiditis: clinical characteristics and treatment outcome in fifty-six consecutive patients diagnosed between 1999 and 2005. J Endocrinol Invest 30: 631-635, 2007.

6. Erdem N, Erdogan M, Ozbek M, et al. Demographic and clinical features of patients with subacute thyroiditis: results of 169 patients from a single university center in Turkey. J Endocrinol Invest 30: 546-550, 2007.

7. Dulipsingh L, Ikram Z, Malchoff CD, Shoukri KC. A cluster of cases of subacute and silent thyroiditis in the northern Connecticut, Greater Hartford area. Conn Med 62: 395-397, 1998.

8. Martino E, Buratti L, Bartalena L, et al. High prevalence of subacute thyroiditis during summer season in Italy. J Endocrinol Invest 10: 321-323, 1987.

9. Saito S, Sakurada T, Yamamoto M, Yamaguchi T, Yoshida K. Subacute thyroiditis: observations on 98 cases for the last 14 years. Tohoku J Exp Med 113: 141-147, 1974.

10. Bech K, Lumholtz B, Nerup J, et al. HLA antigens in Graves' disease. Acta Endocrinol (Copenh) 86: 510-516, 1977.

11. Tamai H, Goto H, Uno H, et al. HLA in Japanese patients with subacute (De Quervain's) thyroiditis. Tissue Antigens 24: 58-59, 1984.

12. Yeo PP, Chan SH, Aw TC, et al. HLA and Chinese patients with subacute (De Quervain's) thyroiditis. Tissue Antigens 17: 249-250, 1981.

13. Kabalak T, Ozgen AG. Familial occurrence of subacute thyroiditis. Endocr J 49: 207-209, 2002.

14. Kramer AB, Roozendaal C, Dullaart RP. Familial occurrence of subacute thyroiditis associated with human leukocyte antigen-B35. Thyroid 14: 544-547, 2004.

15. Hamaguchi E, Nishimura Y, Kaneko S, Takamura T. Subacute thyroiditis developed in identical twins two years apart. Endocr J 52: 559-562, 2005.

16. Yamamoto M, Saito S, Sakurada T, et al. Recurrence of subacute thyroiditis over 10 years after the first attack in three cases. Endocrinol Jpn 35: 833-839, 1988.

17. Iitaka M, Momotani N, Ishii J, Ito K. Incidence of subacute thyroiditis recurrences after a prolonged latency: 24-year survey. $\mathrm{J}$ Clin Endocrinol Metab 81: 466-469, 1996.

18. Ito $\mathrm{M}$, Takamatsu $\mathrm{J}$, Yoshida $\mathrm{S}$, et al. Incomplete thyrotroph suppression determined by third generation thyrotropin assay in subacute thyroiditis compared to silent thyroiditis or hyperthyroid Graves' disease. J Clin Endocrinol Metab 82: 616-619, 1997.

19. Kubota S, Amino N, Matsumoto $Y$, et al. Serial changes in liver function tests in patients with thyrotoxicosis induced by Graves' disease and painless thyroiditis. Thyroid 18: 283-287, 2008.

20. Nishihara E, Miyauchi A, Matsuzuka F, et al. Acute suppurative thyroiditis after fine-needle aspiration causing thyrotoxicosis. Thyroid 15: 1183-1187, 2005.

(C) 2008 The Japanese Society of Internal Medicine http://www.naika.or.jp/imindex.html 